\title{
Intelligent Knowledge Management System for Distributed e-Home Healthcare
}

\author{
U-Hou Choi, Jia-Li Ma, Ran Guo, and Ming-Chui Dong
}

\begin{abstract}
With the functions of on-site multi-vital-signs acquisition, real-time transmission, diagnosis and detailed interpretation, an embedded-link e-home healthcare system on mobile devices brings lots of conveniences for prevention and detection of cardiovascular diseases (CVD). Due to the intrinsic source restriction in those devices, a backyard remote uplink, update and synchronize (UUS) system is imperative to manage the uplinked requests from local mobile devices and feedback promptly the update package. To guarantee the efficiency and availability of UUS when facing thousands of concurrent requests and user clients, an intelligent knowledge management system (KMS) becomes the most significant concern. In this paper, a dedicated customized knowledge base (KB) with simplicity, conformity, extensibility, and flexibility is proposed to release the burden on UUS by reduction of unnecessary help requests. In addition, an intelligent $\mathrm{KB}$ update scheme with delta-change and master-slave approach is pioneered to perform automatic rules update with minimum dataflow while ensuring the reliability and safety of overall system. Via the constructed $\mathrm{KB}$, an intelligent diagnosis system is implemented to provide detailed diagnostic results as well as comprehensive pathological warning messages.
\end{abstract}

Index Terms-CVD diagnosis, distributed e-home healthcare, knowledge base design, knowledge management system, rule update.

\section{INTRODUCTION}

The continuously growing death due to cardiovascular diseases (CVD) makes it the leading cause of death over the world. World Health Organization reported that an estimated 17 million people died from CVD in 2008, representing $30 \%$ of all global deaths and will increase to 23 million by 2030 [1]. Although early detection and timely treatment can prevent CVD efficiently, it highly increases demands on healthcare services and burden on hospitals and medical personnel. As a solution, intelligent mobile devices, wireless communication technology, and remote healthcare architectures are introduced to target transforming hospitalrescue-centered healthcare scheme to patient-preventioncentered approach [2]-[8].

Fig. 1 describes the architecture of distributed e-home healthcare platform as presented in [9]. The overall system consists of three sub-systems, namely upstream (hospital

Manuscript received February 28, 2014; revised April 29, 2014. This work is supported by the research committee of University of Macau under grant No. MYRG184 (Y2-L3)-FST11-DMC and Science and Technology Development Fund (FDCT) of Macau Government under Grant No. 018/2009/A1.

U. H. Choi, J. L. Ma, R. Guo, and M. C. Dong are with the Department of Electrical and Computer Engineering, Faculty of Science and Technology, University of Macau, Macau S.A.R., China (e-mail: mb15509@umac.mo, jimohanqiu@hotmail.com,mb25462@umac.mo,mcdong@umac.mo). server), midstream (patient local computers), and downstream (embedded-link devices, e. g. smartphone, tablet, or personal digital assistant). Multi-vital-signs such as electrocardiography (ECG), sphygmogram (SPG), and heart sound (HS) are acquired and transmitted to wireless-linked midstream and/or downstream for further analysis, interpretation, and diagnosis to procure the results of health status and pathological warning messages. Whenever it encounters doubt regarding the results or failed diagnosis caused by insufficient knowledge base (KB) in lower streams, users can request remotely-linked upstream for help by triggering the customized disease-driven assistance system, i.e. the uplink, update and synchronize (UUS) system. The server in upstream is equipped with abundant $\mathrm{KB}$ rules and comprehensive data base (DB) storing all user clients' information, which is able to recognize and feedback individually the absent $\mathrm{KB}$ rules in user client for update.

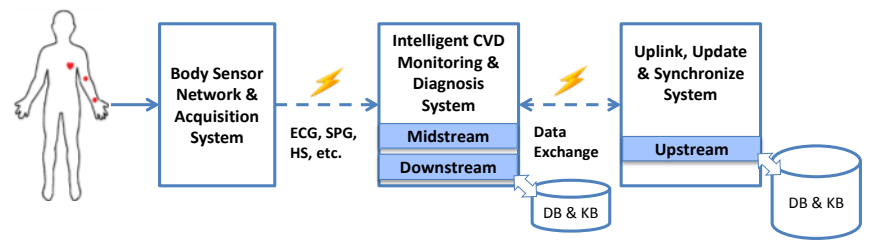

Fig. 1. Systematic architecture of distributed e-home healthcare platform.

Acting as the backend support and remote assistance system, UUS is the key significance to cover the deficiency and limitation in lower streams and satisfy various users' demands, thereby effectively supports the functionality of the overall distributed e-home healthcare system. However, several bottleneck problems must be solved to guarantee the availability and efficiency of UUS:

1) How to store sufficient rules in user mobile devices with limited resources?

2) How to design a customized $\mathrm{KB}$ in user devices and avoid the occurrence of diagnosis failure as much as possible?

3) How to realize $\mathrm{KB}$ update intelligently and automatically?

4) How to handle unexpected circumstances during update?

Tackling these problems, as first attempt among this world so far, a novel intelligent knowledge management system for distributed e-home healthcare application is proposed in this paper. With a well-designed KB framework and customized rules configuration, the resource-limited user devices are able to store adequate personalized knowledge rules, which will speed up the diagnosis, significantly increase the reliability of user client systems, and release the burden on uplinked server by reducing the amount of unnecessary help requests caused by diagnosis failure. Furthermore, an intelligent KB 
delta update scheme is also proposed to perform absent rules update individually and automatically with minimum dataflow, while the pioneered master-slave $\mathrm{KB}$ strategy ensures the reliability and safety of whole system. Finally, an intelligent diagnosis system based on the designed $\mathrm{KB}$ is constructed.

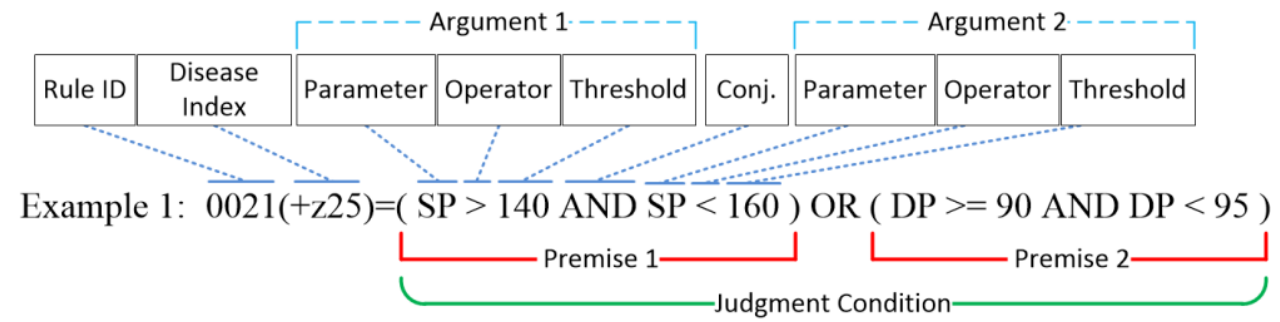

Example 2: $0010(+\mathrm{z} 11)=(\mathrm{VPE}>=\{1.2 *[2 *$ Weight +45$] * 0.0112\})$

Fig. 2. Designed rule structure in cardiovascular diagnostic knowledge base.

TABLE I: CARDIOVASCULAR DISEASES RULES CLASSIFICATION

\begin{tabular}{llll}
\hline \multicolumn{2}{c}{ Name of Package } & \multicolumn{1}{c}{ Description } & Number of Rules \\
\hline \multirow{2}{*}{ Basic Package } & $\begin{array}{l}\text { General rules for preliminary diagnosis of coronary heart diseases, valvular } \\
\text { heart diseases, arrhythmia, cardiac failure, and hypertension }\end{array}$ & 30 \\
\hline & Hypertension & Detailed diagnostic rules for hypertension & 8 \\
\cline { 2 - 4 } $\begin{array}{l}\text { Extension } \\
\text { Packages }\end{array}$ & Pulmonary & Diagnostic rules for pulmonary diseases, including congestion, artery diseases. & 3 \\
\cline { 2 - 4 } & Peripheral & Diagnostic rules for peripheral arterial diseases & 7 \\
\cline { 2 - 4 } & Viscosity & Detailed diagnostic rules for blood viscosity diseases and arteriosclerosis & 6 \\
\cline { 2 - 4 } & Microcirculation & Detailed diagnostic rules for microcirculation diseases & 3 \\
\hline \hline
\end{tabular}

\section{INTELLIGENT KNOWLEDGE MANAGEMENT SySTEM}

The proposed knowledge management system (KMS) consists of three parts. The first part is KB design including $\mathrm{KB}$ framework design and customization; the second part is intelligent $\mathrm{KB}$ update scheme design; the third part is diagnostic system design based on the constructed KB. Since the knowledge management in resource-limited downstream devices is more challenging than in midstream, this paper mainly focuses on the KB update scenario in downstream. Java Development Kit is chosen as the development platform due to its common adaptability on embedded-link devices, whereas the design methodology is also applicable for other software development platforms.

\section{A. Knowledge Base Design}

\section{1) Knowledge base framework}

The design of $\mathrm{KB}$ framework is the foundation of overall KMS and it should satisfy following technical requirements: 1) simplicity to store plenty of knowledge rules in limited file size with easy access format; 2 ) conformity to ensure all the rules in systematic and consistent structure; 3 ) extensibility to perform efficient knowledge update from server; 4) flexibility to better adapt to certain diseases by retrieving specific knowledge.

In the design, $\mathrm{KB}$ rules are written in machine language and stored as text form for easy access and process. As is illustrated in Fig. 2, the structure of KB rule is composed of three parts: rule identification (ID), disease index, and judgment conditions. Each rule starts with a unique ID ranging from 0000 to 9999 . In the construction of disease index, the number following a " $\mathrm{z}$ " corresponds to certain unique disease while symbol "+"/“-"” represents that the following judgment condition is for/against the listed diseases. A rule may contain several disease indices on event that they possess the same condition or symptom. With regard to judgment condition, multiple premises can be defined in the same rule with conjunctions and brackets "( )" added in between. Argument, as the basic component of premises, comprises a real value of certain biomedical parameter calculated from the site-sampled user's vital signs, a threshold value which can be a single number or a curly brackets " \{\} " quoted formula, and an operator to compare the two values. Two rule examples are given in Fig. 2, and we observe that in example 1 two premises are included and connected by a conjunction "OR" in between, whereas the rule in example 2 has only one premise with a formula as threshold value. Different brackets are employed to distinguish among formula, premises, and arguments. The calculating order of those brackets is also considered, i.e. contents within "[ ]" must be calculated prior to those inside " \{\} " and "( )" goes last.

The proposed KB framework has several advantages: 1) straightforward to access with the text format applicable in various mobile devices as well as computers; 2) convenient to synchronize between servers and user clients with a consistent and unique rule ID allocation; 3) effortless to modify and update with an intelligible framework design; 4) resource saving with extremely small $\mathrm{KB}$ file size, e.g. one rule occupies approximately 60-300 bytes with varying complexity, implying that a KB file of 10,000 bytes can accommodate more than 100 rules on average. 


\section{2) Knowledge base customization}

In user client devices, the diagnostic accuracy directly depends on the diversity of local KB rules, yet it is impossible to store all the useful $\mathrm{KB}$ rules in resource-limited mobile devices. Although users can turn to uplinked server for help in case of diagnosis failure and the server will feed back the precise diagnostic results, it is indeed troublesome and inefficient managing thousands of user requests at the same time. Therefore, a customized $\mathrm{KB}$ is imperative to satisfy various users' demands, guarantee reliability of user client systems, as well as release the burden on server through reduction of unnecessary help requests.

For customization, all the useful KB rules are divided into two categories: basic rule package and extension rule packages. The basic package contains all the common CVD diseases while extension packages include several rule packages of different disease categories, such as hypertension package and peripheral arterial disease package. At the startup of e-home healthcare system, each local platform is equipped with identical basic $\mathrm{KB}$ package and can be further customized by downloading various extension rule packages from the server according to user's diagnostic results or request. Table I lists the CVD rules classification applied in our system. With such a tailor-made light KB strategy, the source-restricted mobile devices are capable of providing preliminary diagnostic results for both normal and rare diseases efficiently.

The structure and customization of $\mathrm{KB}$ together strike a subtle balance between computation complexity and diagnostic accuracy, Hence, it not only adapts the source-limited embedded-link devices to diverse users but also dramatically reduces the occurrence of local devices diagnostic failure caused by absent relevant KB rules.

\section{B. Intelligent Knowledge Base Update Design}

\section{1) Delta update scheme}

In event of diagnosis failure caused by absent or outdated rules and demands for KB customization, users' may trigger the $\mathrm{KB}$ update process to seek help from the server. The typical flowchart of local KB update process in client device is depicted in Fig. 3. Firstly, a manual help request is transmitted to server to establish up-down communication link. Afterwards, the whole update process will carry on automatically and intelligently without manual intervention. Local data including acquired biomedical parameters this time, user identification, and other related limited information will be delivered to server for further analysis. Since the server in upstream is equipped with comprehensive data base (DB) storing all user clients' information and abundant disease knowledge, it is able to recognize the rule configuration in user client device. Grounded on this, the server will perform analysis on the received data to obtain detailed highly precise diagnostic results as well as the related rules for diagnosing such a CVD. Finally, the diagnostic results and related useful rules will be packaged and transmitted to user device for local update. Note that, rather than sending back all the useful rules, only the local absent or outdated rules for certain disease are dug out. With such a delta update scheme, it minimizes the necessary dataflow between up and down streams as well as contributes to the fluent execution of local update without the need of re-install.

\section{2) Master-slave knowledge base scheme}

During the $\mathrm{KB}$ update process, several unexpected situations may occur, such as sudden power off, system failure, system exit or etc., which will not only influence the success of update but also damage the original KB file and even the overall system. Tackling this, a master-slave KB update scheme is pioneered in this paper. As depicted in Fig. 3 , on receiving update package from upstream, the local embedded-link device will create a new blank slave KB. Instead of directly overwriting the master $\mathrm{KB}$, the local device will analyze the received update package and original master KB file and write the up-to-date rules into slave $\mathrm{KB}$ file. Before completion of update process, the master $\mathrm{KB}$ is employed for local diagnosis. Once interruption happens during update, the incomplete slave $\mathrm{KB}$ file will be discarded at the beginning of next retrial. After successful update, the slave $\mathrm{KB}$ will be adopted as master $\mathrm{KB}$, while the original master $\mathrm{KB}$ will be archived within the local device for backup. Note that the backed-up KB is to roll back to last version if any abnormality is detected after update or user would like to withdraw the update. All in all, this master-slave update scheme assures safety and reliability of the update process in UUS as well as robustness of the overall system.

\section{Intelligent Diagnosis Program}

Based on the constructed KB, a CVD diagnosis system is designed and constructed. With an intelligent inference engine, the system is able to perform preliminary CVD diagnosis on embedded-link local devices.

First, all the rules in local KB are loaded into inference engine, and then the engine will judge conditions of those rules according to acquired biomedical parameters. If certain rule is satisfied, the corresponding disease index will be recorded as potential disease. According to the inference results, user's health status and possible diseases results as well as the corresponding pathological warning messages will be indicated. The warning messages actually are recommendations for users on timely actions, which may include one or multiple of the following items: 1) improve practices in daily life and diets; 2) pay attention to particular biomedical parameters, non-measurable symptoms or feelings (like dizziness or hard to breath); 3) trigger $\mathrm{KB}$ update for further precise analysis; 4) seek for medical treatment immediately. Fig. 4 shows an example of diagnostic result and warning message is showed on mobile device for a patient with hypertension. As suggested in the warning message, the parameter of blood pressure should be monitored from time to time. Besides, the hypertension extension package is also listed in this interface for customized update. In conclusion, the intelligent diagnostic program provides comprehensive health status information to help users monitor health status and take appropriate actions 
accordingly.

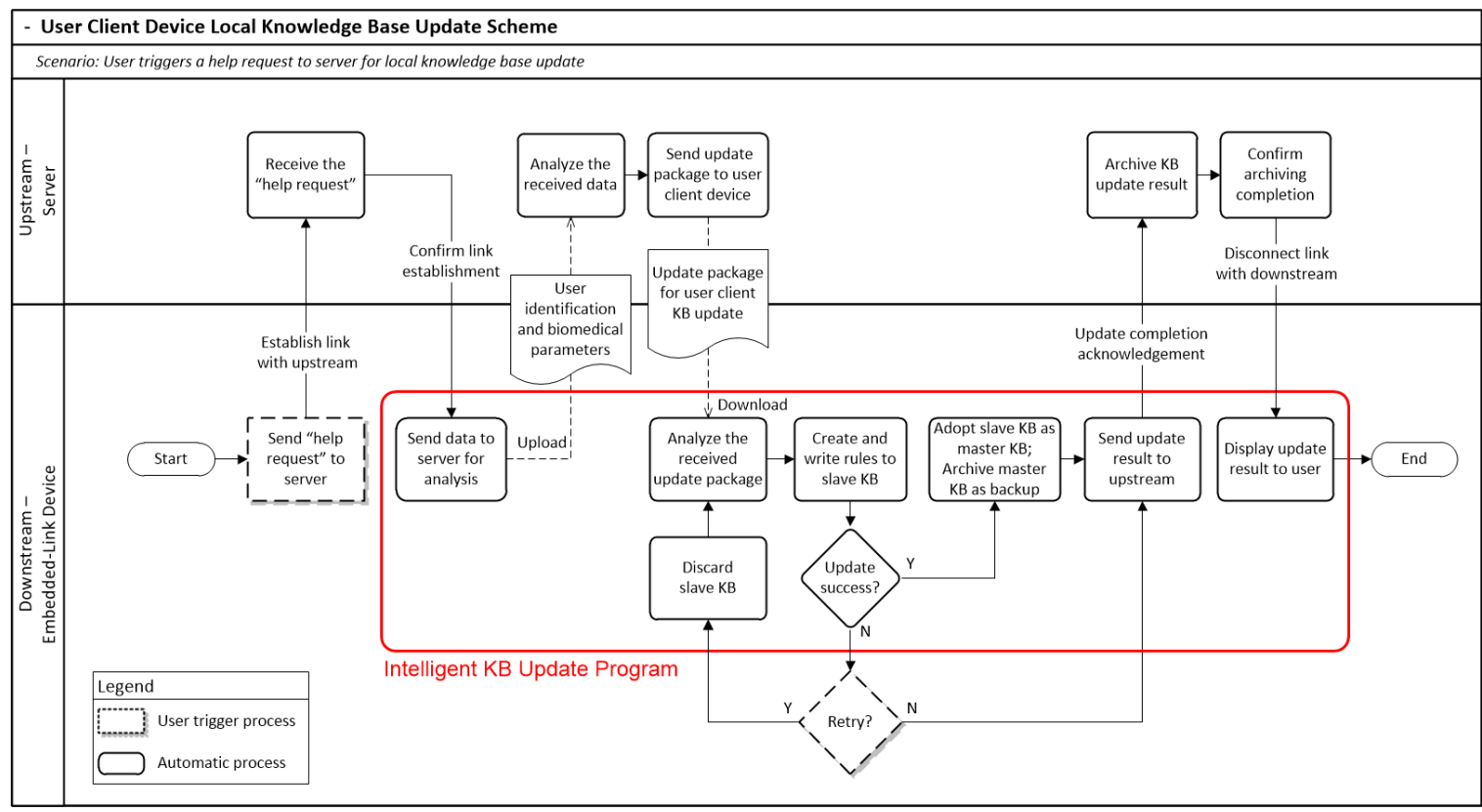

Fig. 3. Flowchart of local KB update process in user client device.

\begin{tabular}{|c|}
\hline Diagnostic Result \\
\hline Diagnostic Message \\
\hline $\begin{array}{l}\text { Arterial hypertension, when blood pressure } \\
\text { sustains over } 160 / 95 \mathrm{~mm} H \mathrm{H} \text { for multiple } \\
\text { measurements without antihypertensive drugs } \\
\text { involved, is the most common cardiovascular } \\
\text { disease phenomenon. Early lesions can lead to } \\
\text { atherosclerosis and thrombosis, late period } \\
\text { will cause damage to heart, brain, kidneys and } \\
\text { other vital organs. This is a major risk factor for } \\
\text { coronary heart disease and stroke. It is } \\
\text { recommended to have regular measurement } \\
\text { of blood pressure, keep monitoring of health } \\
\text { status with this system and seek for timely } \\
\text { medical treatment in case of deterioration. }\end{array}$ \\
\hline Recommended Actions \\
\hline Keep monitoring blood pressure \\
\hline Suggested KB Update Options \\
\hline Hypertension Extension Package (DOWNLOAD) \\
\hline More... \\
\hline
\end{tabular}

Fig. 4. A diagnostic result of hypertension shown on mobile device.

\section{CONCLUSION}

In this paper, a novel intelligent KMS is proposed and developed for distributed e-home healthcare applications via Java language. With a dedicated KB framework and customized rules configuration, the resource-limited user mobile devices are able to store adequate personalized knowledge rules. An intelligent $\mathrm{KB}$ delta update scheme is introduced to perform absent rules update individually and automatically with minimum dataflow. Furthermore, the pioneered master-slave KB approach ensures the reliability and safety of the whole system. Finally, an intelligent diagnosis system with the designed KB is constructed. The future work will be the system improvement in terms of multi-tasking and prioritizing of user requests, simplifying knowledge rules representation and fastering the diagnostic procedure with higher diagnostic accuracy.

\section{REFERENCES}

[1] World Health Organization, Global Status Report on Noncommunicable Diseases 2010, Geneva, Switzerland: World Health Organization, 2011, ch. 1, pp. 9-11.

[2] U. Varshney, "Pervasive healthcare and wireless health monitoring," Mobile Networks and Applications, vol. 12, no. 2-3, pp. 113-127, Mar. 2007.

[3] F. Kart, L. E. Moser, and P. M. Melliar-Smith, "Building a distributed e-healthcare system using SOA," IEEE IT Professional, vol. 10, no. 2, pp. 24-30, Mar. 2008.

[4] F. Sufi, Q. Fang, and S. Mahmoud, "Novel methods of faster cardiovascular diagnosis in wireless telecardiology," IEEE J. Sel. Areas Commun., pp. 537-552, vol. 27, no. 4, Apr. 2009.

[5] M. Rusu, G. Saplacan, G. Sebestyen, C. Cenan, L. Krucz, T. Nicoara, and N. Todor, "Distributed e-health system with smart self-care units," in Proc. IEEE Int. Conf. on Intelligent Computer Communication and Processing, 2009, pp. 307-314.

[6] D. Rehunathan, S. Bhatti, O. Chandran, and H. Pan, "vNurse: Using virtualisation on mobile phones for remote health monitoring," in Proc. IEEE Int. Conf. on e-Health Networking Applications and Services, 2011, pp. 82-85.

[7] M. Bajorek and J. Nowak, "The role of a mobile device in a home monitoring healthcare system," in Proc. Federated Conference on Computer Science and Information Systems, 2011, pp. 371-374.

[8] R. Malmathanraj and M. Arun, "A distributed e-healthcare system for patient monitoring and diagnosis," in Proc. IEEE Int. Conf. on Advanced Communication Control and Computing Technologies, 2012, pp. 71-77.

[9] J. L. Ma, R. Guo, U. H. Choi, and M. C. Dong, "A framework architecture for versatile distributed e-home healthcare," presented at the 9th FTRA Int. Conf. on Future Information Technology, Zhangjiajie, China, May 28-31, 2014.

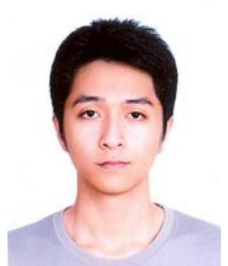

U-Hou Choi received his B.Sc. degree in electrical and electronics engineering from University of Macau in 2009. He is currently a master student in EEE at University of Macau. His main research interests are system automation, AI and its application in biomedical engineering. 


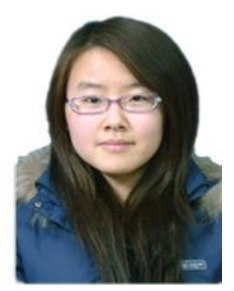

Jia-Li Ma received the B.Sc. degree in electrical and information engineering from Tianjin University, Tianjin, China in 2011. Currently, she is pursuing her master degree in electrical and computer engineering at University of Macau. Her research interests include biomedical signals compression and construction of embedded-link e-health systems.

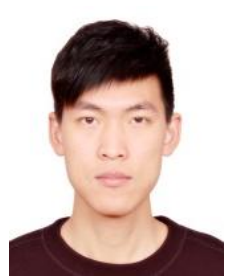

Ran Guo received the B.Sc. degree from Macau University of Science and Technology in 2012. He is currently a master student in electrical and computer engineering, University of Macau. His research interests are mainly about information technology, network analysis, expert system and biomedical signal processing.

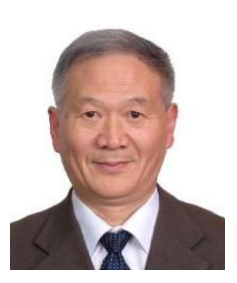

Ming-Chui Dong received his M.Sc. degree in EEE in 1975 at Tsinghua University, China. He is a visiting scholar in EEE in 1981 at Rome University, Italy. He is a full professor and a PhD supervisor of ECE Department of University of Macau, Automation Department of Tsinghua University, China and professor of Yan Tai University, China. His main research interests are AI and its application in biomedical engineering, CIMS,

fault diagnosis, AI in vision-to-text, voice-to-text machine translation etc. 